\title{
Prendre et/ou porter les armes entre les XIIIe et XIXe siècles, un facteur de politisation ? Quelques perspectives
}

Taking up and/or bearing arms, betweens the 13th and the 19th centuries: a politicizing factor? A few perspectives

\section{Yann Lagadec}

\section{(2) OpenEdition}

Journals

\section{Édition électronique}

URL : http://journals.openedition.org/abpo/2234

DOI : $10.4000 /$ abpo.2234

ISBN : 978-2-7535-1841-4

ISSN : 2108-6443

\section{Éditeur}

Presses universitaires de Rennes

\section{Édition imprimée}

Date de publication : 30 décembre 2011

Pagination : 7-20

ISBN : 978-2-7535-1839-1

ISSN : 0399-0826

Référence électronique

Yann Lagadec, "Prendre et/ou porter les armes entre les XIIle et XIXe siècles, un facteur de politisation? Quelques perspectives ", Annales de Bretagne et des Pays de l'Ouest [En ligne], 118-4 | 2011, mis en ligne le 30 décembre 2013, consulté le 19 avril 2019. URL : http:// journals.openedition.org/abpo/2234; DOI : 10.4000/abpo.2234 


\title{
Prendre et/ou porter les armes entre les XIII ${ }^{\mathrm{e}}$ et XIX ${ }^{\mathrm{e}}$ siècles, un facteur de politisation? Quelques perspectives
}

\author{
Yann LAGADEC \\ Maître de conférences en histoire moderne \\ Université Rennes 2 Haute-Bretagne - CERHIO, UMR CNRS 6258
}

La question des liens entre la politisation et la prise d'armes dans les sociétés des XIII ${ }^{\mathrm{e}}$-XIX ${ }^{\mathrm{e}}$ siècles n'est sans doute pas totalement neuve ${ }^{1}$. Rappelons qu'elle se trouvait, au moins implicitement, au cœur de nombre des contributions au colloque organisé il y a désormais plus de 25 ans par J. Nicolas sur Mouvements populaires et conscience sociale ${ }^{2}$. Plus récemment, la question de la prise d'arme - populaire et urbaine pour l'essentiel - a été largement abordée lors du colloque sur la garde nationale organisé à Rennes par S. Bianchi et R. Dupuy ${ }^{3}$. Le même R. Dupuy, dans ses recherches sur la chouannerie d'une part, sur la " politique du peuple " d'autre part, a ouvert des pistes particulièrement stimulantes - mais aussi, parfois, sujettes à critique ou nuance - concernant la prise d'armes populaire et ses significations politiques, notamment au moment de la Révolution française ${ }^{4}$. Et l'on pourrait, bien évidemment, multiplier les exemples à l'envi, des récents travaux de L. Hincker sur la garde nationale parisienne aux réflexions, plus anciennes mais toujours éclairantes, d'Y.-M. Bercé sur Les soulèvements paysans en France du XVe au XIX siècle ${ }^{5}$.

1. Ce texte ne serait pas ce qu'il est sans les suggestions de Philippe Hamon, Jean Le Bihan et Samuel Leturcq. Qu'ils en soient ici remerciés.

2. NICOLAS, Jean (dir.), Mouvements populaires et conscience sociale (XVI-XIXe siècles), Actes du colloque de Paris (1984), Paris, Maloine, 1984.

3. BIANCHI, Serge et DuPUY, Roger (dir.), La Garde nationale : entre peuple et nation en armes, Rennes, PUR, 2006.

4. DuPuY, Roger, De la Révolution à la chouannerie. Paysans en Bretagne, 1788-1794, Paris, Flammarion, 1988 et Dupuy Roger, La Politique du peuple. Racines, permanences et ambiguïtés du populisme, Paris, Albin Michel, 2002.

5. HINCKER, Louis, Citoyens combattants. Paris, 1848-1851, Lille, Presses universitaires du Septentrion, 2008 et BercÉ, Yves-Marie, Croquants et Nu-Pieds. Les Soulèvements paysans en France du XVI au XIXe siècle, Paris, Gallimard-Julliard, 1974. 
Pourtant, il nous a semblé qu'il était nécessaire d'affiner encore les questionnements sur les rapports entre la prise d'armes - sous toutes ses formes, légale ou non, encadrée ou pas - et la politisation, une réflexion engagée dans le cadre plus global d'un programme de recherche de l'Agence nationale de la recherche (ANR) consacré aux liens entre "Conflits et politisation ${ }^{6} "$.

\section{Autour de la notion - trop - plastique de " politisation "...}

Au-delà des quelques grands moments que sont, en France principalement, la Révolution, les Trois Glorieuses, les journées de Février et de Juin ou la Commune, la question des liens entre la prise d'armes et la politisation reste bien plus ouverte qu'il n'y paraît.

La première dimension du débat porte sur la notion même de " politisation ", mais aussi des rapports entre ce que l'on a pris l'habitude de qualifier de "politisation " et la politique... ou le politique. La chose vaut tout particulièrement pour ce qui concerne les milieux populaires et, plus encore d'ailleurs, les milieux populaires ruraux, tant semble aller de soi la " politisation " des élites sociales et culturelles constituant une " opinion publique " aux contours pourtant tout aussi mal définis ${ }^{7}$. Apolitisme - voire indifférence et ignorance - en deçà de la Révolution, " politique " au-delà : ainsi pourrait-on, certes grossièrement, résumer l'essentiel de l'historiographie consacrée à ces questions, une historiographie encore largement inspirée par une définition de ce terme reposant sur les deux inébranlables piliers que seraient l'intégration aux débats nationaux et les élections ${ }^{8}$. Encore ce terminus a quo que constituerait la décennie révolutionnaire n'est-il accepté que par certains, historiens de la Révolution pour la plupart ${ }^{9} .1848$ et l'introduction du suffrage universel masculin en ce qui concerne la France, ou des dates en tous points comparables dans les pays voisins marqués par l'émergence concomitante d'une certaine citoyenneté électorale et de partis politiques plus structurés sont finale-

6. Ce programme, lancé en 2007 par Laurent Bourquin et Philippe Hamon, professeurs d'histoire moderne à l'Université du Maine pour le premier, à l'Université de Rennes 2 pour le second, s'est traduit par l'organisation de plusieurs colloques et journées d'études. Les textes qui suivent sont ceux des communications de l'une de ces journées, tenue à Rennes le 9 janvier 2009 .

7. Sur cet aspect, nous renvoyons à Bourquin, Laurent, HAMON, Philippe, KARILA-COHEN, Pierre et Michon, Cédric (dir.), Conflits et opinion(s). Actes du colloque du Mans de mai 2009, Rennes, Presses universitaires de Rennes, à paraître en 2011.

8. Sur ce point, se reporter aux pratiques synthèses historiographiques de PÉcouT, Gilles, "La politisation des paysans au XIX ${ }^{\mathrm{e}}$ siècle. Réflexions sur l'histoire politique des campagnes françaises ", Histoire et sociétés rurales, n², 1994, p. 91-125 et de HINCKER, Louis, "La politisation des milieux populaires en France au XIX siècle : constructions d'historiens. Esquisse d'un bilan (1948-1997) ", Revue d'histoire du XIXe siècle, 1997-1, p. 89-105.

9. C'est le cas, entre autres, de Vovelle, Michel, La Découverte de la politique. Géopolitique de la Révolution française, Paris, La Découverte, 1992 ou encore de BianchI, Serge, La Révolution et la Première République au village. Pouvoirs, votes et politisation dans les campagnes d'Île-de-France, 1787-1800, Paris, CTHS, 2003. 
ment bien plus souvent retenues par les spécialistes du XIX siècle $^{10}$. Parce que la politisation ne serait que l'" inculcation de la démocratie " comme le pense M. Agulhon, indissociablement liée à "l'élection concurrentielle " selon P. Rosanvallon et à la " lutte partisane autour de l'élection " pour M. Crozier et E. Friedberg ${ }^{11}$, non seulement la question de ses rapports avec la prise d'arme n'aurait guère de sens, mais encore son extension à des périodes antérieures à la Révolution confinerait par certains aspects à l'absurde.

Depuis une trentaine d'années en effet, certains historiens - modernistes notamment - se sont attachés à dépasser une vision par trop réductrice de la politique, celle opposant d'une part une modernité, socialement ancrée dans les élites urbaines, centrée sur des débats nationaux, et d'autre part un archaïsme populaire et, bien plus encore, rural et paysan, fait d'indifférence et de soumission à des notables traditionnels ou à un clergé tout puissant, tout particulièrement - mais pas seulement - dans une vaste France de l'Ouest ${ }^{12}$. En simplifiant, pour les tenants de ce courant, l'histoire de l'apprentissage de la politique - ici entendue comme la lutte pour la conquête ou la conservation du pouvoir, à quelque niveau

10. Agulhon, Maurice, La République au village. Les populations du Var de la Révolution à la Seconde République, Paris, Seuil, 1979 constitue l'ouvrage emblématique de cette conception. WEBER, Eugen, La Fin des terroirs. La modernisation de la France rurale (18701914), Paris, Fayard, 1983 et BERGER, Suzanne, Les Paysans contre la politique. L'organisation rurale en Bretagne (1911-1974), Paris, Seuil, 1975, au titre très révélateur, considèrent comme plus tardive encore la politisation du monde rural, la situant dans les années 1880-1914. McPheE, Peter, Les Semailles de la République dans les Pyrénées-Orientales (1846-1852) : classes sociales, culture et politique, Perpignan, Les Publications de l'Olivier, 1995 ou GuIONNET, Christine, L'Apprentissage de la politique moderne. Les élections municipales sous la monarchie de Juillet, Paris, L'Harmattan, 1997, sur des points très différents, conteste pour l'un, nuance pour l'autre ces différentes approches, situant la politisation des campagnes en amont de 1848. Plus globalement, le faible nombre d'études consacrées à la vie politique - et notamment à la vie politique locale - entre 1800 et 1848 atteste en négatif la polarisation du débat autour des deux moments-clés que constitueraient la Révolution et les années 1848.

11. AgulHon, Maurice, " 1848 , le suffrage universel et la politisation des campagnes françaises ", dans : Agulhon, Maurice, Histoire vagabonde, III : La Politique en France, d'hier à aujourd'hui, Paris, Gallimard, p. 79; Rosanvallon, Pierre, "Introduction ", dans : GuIONnET, Christine, L'Apprentissage de la politique moderne. Les élections municipales sous la monarchie de Juillet, Paris, L'Harmattan, 1997, p. IV; CROZIER, Michel et FRIEDBERG, Erhard, L'Acteur et le système. Les contraintes de l'action collective, Paris, Seuil, 1977, p. 260.

12. Citons, sans prétention à l'exhaustivité, les travaux de DuPuY, Roger, De la Révolution à la Chouannerie. Paysans en Bretagne (1788-1794), Paris, Flammarion, 1988, SouRIAC, René, "Les paysans et la politique aux XVI $\mathrm{x}^{\mathrm{e}} \mathrm{xVII}{ }^{\mathrm{e}}$ siècles ", Histoire et sociétés rurales, $\mathrm{n}^{\circ} 3$, 1995-1, p. 117-122, JESSENNE, Jean-Pierre, « Du sujet au citoyen. La participation rurale aux affaires publiques de l'Ancien Régime au Consulat ", Histoire et sociétés rurales, ${ }^{\circ}$ 3, 19951, pp. 123-132 et JESSENNE, Jean-Pierre, "Synergie nationale et dynamique communautaire dans l'évolution politique rurale par-delà la Révolution française (vers 1780-vers 1830) ", dans : AgulHon, Maurice (dir.), La Politisation des campagnes au XIX siècle (France, Italie, Espagne Portugal et Grèce). Actes du colloque de Rome (20-22 février 1997), Rome, EFR, 2000, p. 57-79. 
que ce soit ${ }^{13}$ - ne saurait être uniquement celle d'une acculturation, de l'imposition d'une nouvelle culture, politique en l'occurrence, celle des élites, à des " demi-sauvages ». L'étude du processus d' "imposition d'une bonne manière de faire de la politique ", selon l'expression d'A. Collovald et et F. Sawicki, ne saurait donc, à elle seule, épuiser l'histoire politique des milieux populaires, urbains comme ruraux, la politisation ne se limitant pas alors à la "descente de la politique vers les masses " évoquée naguère par $\mathrm{M}$. Agulhon ${ }^{14}$; elle inclurait toute une série de phénomènes plus complexes.

L'armement populaire, la prise d'arme font indéniablement partie de ces phénomènes. La chose semble évidente en ce qui concerne la Fronde par exemple, notamment pour le peuple parisien; mais elle ne saurait être réduite au seul monde urbain. Comment analyser, par exemple, l'affrontement, en 1589, entre les paysans du " party de la Ligue " et ceux du "party du Roy ", au moment du siège de Vitré d'une part, entre avril et août, d'autre part autour de la petite ville de Châteaugiron, attaquée, prise et reprise à une dizaine d'occasions entre le printemps et l'automne, notamment par ces communes ou " milices " rurales, royalistes pour certaines, ligueuses surtout ${ }^{15}$ ? Comment ne pas voir, dans les cris de "Vive le Roy sans gabelle ", lancés par les révoltés quercynois en 1707, les traces d'une politisation ${ }^{16}$ ? Et quel sens, autre que politique, donner à la représentation de leurs maires en armes sur leur sceaux par les communes, y compris rurales, du Ponthieu dès le XIII ${ }^{\mathrm{e}}$ siècle, en une zone - sans doute n'est-ce pas un hasard - disputée entre France et Angleterre?

13. Je fais mienne ici la définition proposée par Philippe Braud dans GrAWITZ, Madeleine et LECA, Jean (dir.), Traité de science politique. Tome 1, Paris, PUF, 1985, p. 389-390, directement inspirée de celle de M. Weber pour qui la politique est "l'ensemble des efforts que l'on fait en vue de participer au pouvoir ou d'influer sur la répartition du pouvoir ". Celle-ci permet en effet de s'affranchir, d'emblée, du débat sur l'archaïsme et/ou la modernité de telle ou telle pratique. Elle est, de ce fait, proche de celle adoptée par nombre d'anthropologues en ce qu'elle offre la possibilité de ne pas se limiter au " modèle " occidental de la démocratie libérale.

14. Collovald, Annie et SAWICKI, Frédéric, « Le populaire et le politique : quelques pistes de recherche en guise d'introduction ", Politix, n 13, 1991, p. 7-19 et Agulhon, Maurice, La République au village..., op. cit. Il convient d'ailleurs de noter ici la distinction peu à peu apparue entre l'étude - que l'on pourrait qualifier de "traditionnelle " - de la vie politique dans un département et celle, inaugurée par M. Agulhon, des modalités de la politisation des masses rurales.

15. Sur cet épisode, voir les travaux complémentaires de LAGRÉE, Michel, « La structure pérenne. Événement et histoire en Bretagne orientale ( $\mathrm{XVI}^{\mathrm{e}}-\mathrm{xx}{ }^{\mathrm{e}}$ siècles) ", Revue d'histoire moderne et contemporaine, 1976-3, p. 394-407, LAGADEC, Yann, " Les communes rurales de Haute-Bretagne pendant la Ligue (1589) : une préfiguration des gardes nationales (1789)? ", dans BIANCHI, Serge et DuPUY, Roger (dir.), La Garde nationale ..., op. cit., p. 27-39 et HAMON, Philippe, " "Vitray, qui s'en alloit perdu..." (Brantôme). Le siège de Vitré et les engagements militaires en Haute-Bretagne au début des guerres de la Ligue (mars-août 1589) ", Mémoires de la Société d'histoire et d'archéologie de Bretagne, 2009, p. 111-152.

16. BERCÉ, Yves-Marie, Croquants et Nu-Pieds..., op. cit., p. 75. 
Le dossier qui suit se veut, pour une part au moins, une participation à la réflexion sur la qualification de telles pratiques. Faut-il y voir les traces d'une " subculture politique " ou d'une " proto-politique " à l'instar de ce que proposait R. Dupuy en $1997^{17}$ ? Peut-on considérer qu'elles relèvent de la "politique informelle " au cœur des réflexions d'un récent colloque ${ }^{18}$ ? Nous ne trancherons pas ici, si ce n'est pour affirmer le caractère éminemment politique de la prise d'arme.

\section{Prendre et porter les armes entre XIII ${ }^{\mathrm{e}}$ et $\mathrm{XIX}^{\mathrm{e}}$ siècles}

Il convient, avant toute chose, de prendre soin de distinguer un certain nombre de réalités a priori assez proches, de préciser aussi ce que fut notre démarche ici.

La première des distinctions à faire concerne port d'armes et prise d'armes. Alors que l'un relève très largement d'un certain quotidien et, dans une large mesure, d'une certaine légalité, l'autre nous renvoie sans doute à des situations plus exceptionnelles, dans lesquelles la légitimité revendiquée supplée souvent à la légalité : l'on ne prend les armes que lorsque les circonstances l'imposent, en général dans le cadre de la révolte ou pour se prémunir contre les conséquences de la guerre. Pour autant, la " politisation " n'est pas propre à la seule prise d'armes : la participation active à la garde nationale pendant la Révolution ou la monarchie de Juillet en France, l'intégration à un corps de volunteers en Irlande au tournant des années 1770-1780, en Grande-Bretagne durant les French Wars, de 1793 à 1815 , en témoignent par exemple ${ }^{19}$. Porter les armes, tout comme les prendre, revient à affirmer son statut d'homme libre, parfois de citoyen, à dire aussi son appartenance à une communauté, rurale ou urbaine, et sa volonté de participer à la gestion de ses affaires et à sa protection active. L'attachement, en plein $\mathrm{XVIII}^{\mathrm{e}}$ siècle, de nombre de villes françaises - et de l'Ouest en particulier - au maintien d'une milice bourgeoise pourtant peu active le dit bien : les problèmes de recrutement, la difficulté à réunir les hommes devant s'entraîner régulièrement n'empêchent pas la volonté sans

17. DuPuY, Roger, « Les campagnes blanches de l'Ouest de la France (1793-1850) : subculture politique ou protopolitique populaire? ", dans : AGULHON, Maurice (dir.), La Politisation des campagnes..., op. cit., p. 343-350.

18. Organisé les 16-17 décembre 2009 à l'Université de Bretagne-Sud (Lorient) par Michel Offerlé, François Ploux et Laurent Le Gall, ce colloque portait sur " La politique informelle en France et en Europe $\left(19^{\mathrm{e}}-21^{\mathrm{e}} \mathrm{s}\right.$.)".

19. Sur le rôle des Irish Volunteers et des Volunteers dans la politisation des milieux populaires irlandais et britanniques, qui bénéficient d'une abondante bibliographie, nous renvoyons à BARTLETT, Thomas " Militarization and Politicization in Ireland 1780-1820", dans : BERgEROn, Louis et Cullen, Louis M. (dir.), Culture et pratiques politiques en France et en Irlande, XVIe-XVIII siècles. Actes du colloque de Marseille, 28 septembre-2 octobre 1988, Paris, Centre de Recherches Historiques, 1991, p. 125-136, O'SNODAIGH, Patraig "The Volunteers of 82 : A Citizen Army or Armed Citizens. A Bicentennial Retrospective ", Irish Studies, Vol. XV, n 60, 1983, p. 177-188 ou CooKson, J. E., " The English Volunteer Movement of the French Wars, 1793-1815 : Some Contextes ", Historical Journal, 1989, Vol. XXXII, p. 867-891. 
cesse réaffirmée de conserver cette institution héritée du Moyen Âge ${ }^{20}$. Tout aussi significatif est, de ce point de vue, le fait que les capitaines de la milice de Nouvelle-France aient continué à entraîner leurs hommes après la chute de Montréal, avec l'autorisation des Britanniques contre lesquels ils combattaient quelques mois plus tôt, auxquels ils avaient remis leurs armes au printemps 1760 et prêté serment d'allégeance ${ }^{21}$.

La seconde distinction que nous avons souhaité opérer est celle entre les forces armées régulières - royales ou nationales - et, celles, de " circonstance ", constituées par les milices en tous genres. La question de la politisation des premières est en effet de nature largement différente, dans la mesure où elle ne se pose qu'en quelques situations très particulières. Il n'est point question de politisation, par exemple, des armées françaises - ou prussiennes - durant les guerres de Succession d'Espagne, de Pologne, d'Autriche, ou durant la guerre de Sept Ans pour n'en rester qu'à ces quelques exemples. Il en va différemment pendant la Révolution en France, au printemps et à l'été 1789, alors que les pamphlets et libelles signés de militaires favorables aux idées nouvelles se multiplient, alors que s'affrontent parfois, comme à Paris le 12 juillet, régiments acquis au tiers-état et unités restées fidèles à la monarchie. Une situation qui n'évolue guère dans les mois qui suivent : l'on retiendra, sans prétention à l'exhaustivité, les événements de Lille en avril 1790, l'affaire de Nancy en août de la même année, les troubles militaires du printemps 1791 à Aix et Marseille ou les mutineries de matelots des vaisseaux du port de Brest en septembre-octobre 1790 puis en $1792^{22}$. En Angleterre et, de manière

20. On trouvera des exemples dans AuBERT, Gauthier, " Devenir officier dans la milice bourgeoise de Rennes sous l'Ancien Régime ", dans : BIANCHI Serge et DupuY Roger (dir.), La Garde nationale..., op. cit., p. 59-71, dans PERREON, Stéphane, " D'un rôle militaire à une fonction sociale : les milices bourgeoises de Bretagne dans la seconde moitié du XVIII ${ }^{\mathrm{e}}$ siècle ", Ibid., p. 95-109 ou encore dans BAUMIER, Béatrice, " De la milice bourgeoise à la Garde nationale : l'exemple de Tours des années 1760 à 1792 ", Ibid., p. 113-126.

21. WARD, Matthew, The Battle for Quebec, 1759, Stroud, Tempus, 2009, p. 236.

22. Sur ces aspects, nous renvoyons aux travaux récents de FORREST, Alan, Soldiers of the French Revolution, Durham/Londres, Duke University Press, 2003 ou HIPPLER, Thomas, Soldats et citoyens. Naissance du service militaire en France et en Prusse, Paris, PUF, 2006. La question d'une politisation particulière des forces armées ressurgit périodiquement en France après la décennie révolutionnaire : politisation républicaine hostile à l'évolution du régime consulaire dans les années 1800-1804, républicaine et bonapartiste dans les premières années de la Restauration notamment, carliste pendant la monarchie de Juillet, monarchiste pendant les premières années de la République opportuniste. Le cas de la gendarmerie - notamment dans l'Ouest de la France - a été particulièrement bien éclairé par LIGNEREUX Aurélien, " De la chouannerie à la gendarmerie? Les captations partisanes de la force publique dans les départements de l'Ouest (1814-1832) ", Annales de Bretagne et des Pays de l'Ouest, 114/2, 2007, p. 109-119, Luc Jean-Noël, " La transition politique, observatoire régional de la gendarmerie du XIX ${ }^{\mathrm{e}}$ siècle ", Annales de Bretagne et des Pays de l'Ouest, 114/2, 2007, p. 185-194 et PÉNIGUEL Jean-François, "Les épurations dans la gendarmerie en Ille-et-Vilaine sous la Seconde Restauration ", Revue historique des armées, 215, 1999, p. 117-128 ou encore, sous forme de synthèse, par HouTE, Arnaud-Dominique, "Une question de loyauté. Les épurations de la gendarmerie (1791-1939) », dans : BERGERE, Marc et LE BiHAN, Jean (dir.), Fonctionnaires dans la tourmente. Epurations administratives et 
plus générale, en Grande-Bretagne, le souvenir de la guerre civile et du protectorat de Cromwell qui ont fait de l'armée désormais permanente un enjeu politique de première importance conduit à redouter son éventuelle politisation et de ce fait à la contrôler, au même titre que sa potentielle confessionnalisation d'ailleurs, justifiant tout autant les réticences tardives de certains face à l'idée d'une standing army - une armée permanente - que les purges de 1715-1717 par exemple ${ }^{23}$. Ainsi, initialement perçue comme au service du roi, l'armée passe peu à peu, au cours du XVIII ${ }^{\mathrm{e}}$ siècle, au service du " public ", de l'Etat, impliquant une dépolitisation qui gagne aussi la seconde armée permanente que constitue la Militia, réorganisée en 1757; à défaut d'avoir été expressément proscrits dans l'Army ou la Navy - certains officiers démissionnent par exemple dans les années 1770 par refus de la guerre contre les Insurgents -, l'esprit partisan est ainsi largement mis de côté par une sorte d'accord tacite ${ }^{24}$.

La troisième distinction est celle entre prise d'armes populaire et prise d'armes des élites, nobiliaires notamment, pour reprendre une terminologie qui n'est désormais plus guère usitée mais reste fort pratique. Certes, cette distinction est pour une part artificielle : l'on sait depuis longtemps que certains nobles, certains petits seigneurs, participèrent, par exemple, aux révoltes "populaires " des années centrales du XvII ${ }^{\mathrm{e}}$ siècle, dans le Sud-Ouest de la France notamment et il n'est guère besoin de rappeler ici le rôle des aristocrates contre-révolutionnaires dans l'encadrement - plus que dans le déclenchement - des mouvements paysans anti-révolutionnaires dans l'Ouest de la France. Il n'en reste pas moins que la question de la dimension « politique » des prises d'armes nobiliaires ne semble plus

transitions politiques à l'époque contemporaine, Chêne-Bourg, Georg, 2009, p. 121-142. On trouvera des approches plus générales dans LuTuN, Bernard, "1814-1817 ou l'épuration dans la Marine ", Revue historique, 583, 1992, p. 61-86, MonTAGnE, Pierre-Antoine, Le comportement politique de l'armée à Lyon sous la monarchie de Juillet et la Seconde République, Paris, Librairie générale de droit et de jurisprudence, 1966 ou, plus récemment, dans l'ouvrage de CHANET, Jean-François, Vers l'armée nouvelle. République conservatrice et réforme militaire, 1871-1879, Rennes, Presses universitaires de Rennes, 2006.

23. CHILDS, John, The Army, James II, and the Glorious Revolution, Manchester, Manchester University Press, 1980, p. 56-82, a bien montré, par exemple, comment les promotions dans l'armée de Jacques II - une armée aux effectifs croissants et aux possibilités d'ascension d'autant plus nombreuses - étaient largement liées à un certain conformisme politique et religieux, le roi n'hésitant pas, par exemple, à mettre fin aux commissions de plus de $80 \%$ des officiers de son armée d'Irlande, fournissant indirectement autant de partisans à Guillaume d'Orange. Le XVIII ${ }^{\mathrm{e}}$ siècle voit, par ailleurs, un nombre croissant d'officiers devenir Members of Parliament, ce qui ne va pas sans poser problème : en 1715, six $M P$ 's sont contraints à abandonner leur commission d'officier en raison de leur proximité avec les Tories. En 1789 encore, le marquis de Lothian est démis suite à un vote contraire aux attentes de George III. Sur ces questions politiques, voir la synthèse de STRACHAN, Hew, The Politics of the British Army, Oxford, Oxford University Press, 1997, p. 20-43.

24. CoOKSON, John E., "Service without Politics? Army, Militia and Volunteers in Britain during the American and French Revolutionary Wars ", War in History, 2003-4, p. 381-397 et Cookson, John E., The British Armed Nation, 1793-1815, Oxford, Clarendon Press, 1997, p. 66-94. En Grande-Bretagne, ce sont les volunteers qui sont, à compter de 1793, au cœur des luttes entre partisans de Fox et de Pitt. 
guère se poser à la plupart des historiens : le " devoir de révolte " de la noblesse serait en quelque sorte par essence politique, au contraire de la prise d'armes populaire, réaction irraisonnée facilement suspectée d'archaïsme ${ }^{25}$.

Certes, la dimension politique de l'appel aux armes n'apparaît pas toujours de manière évidente. La mobilisation d'hommes en armes pour combattre le brigandage - réel ou supposé, comme lors de la Grande Peur -, l'épidémie par la mise en place de cordons sanitaires, les loups mais aussi l'ennemi extérieur, à l'instar des paysans flamands de Rumegies qui mettent en déroute une bande de pillards espagnols en 1694 ou des habitants de Verzy, dans la Marne, contre les cosaques en 1814, bref tout ce qui peut apparaître comme mettant en danger la communauté, rurale ou urbaine, pourrait n'avoir qu'un lointain rapport avec une éventuelle politisation ${ }^{26}$. Voire. En donnant à certains la possibilité de s'armer au profit de la collectivité, la prise d'armes, légale ou non, participe indéniablement de la construction - au moins locale - du politique.

Encore faut-il - que l'on pardonne ce truisme - disposer d'armes, à prendre ou à porter.

\section{Prendre et porter les armes : quelles armes?}

Prendre les armes, porter les armes, certes. Mais quelles armes, seraiton tenté d'écrire. Pour évidente qu'elle soit, la question mérite pourtant d'être posée, au moins pour toute une partie de la période considérée, car si le port d'armes est presque constitutif de l'identité nobiliaire, la plus grande partie des habitants en sont exclus.

Passons, sans insister, sur le recours aux principaux arsenaux et dépôts d'armes, pris par la force éventuellement. Dépôts privés tout d'abord, à commencer par ceux des principales maisons du lieu, nobles souvent, bourgeoises et patriotes ailleurs : alors qu'en mars 1793, les insurgés bretons des environs de Vitré "se transportèrent par pelotons chez plusieurs citoyens" - entendre bons citoyens - à la recherche d'armes, les communes de l'Angoumois, en 1636, enjoignaient par exemple " à chacune parroisse de faire marcher tous les gentilzhommes et donner les armes, à peine de brusler leurs maisons et n'estre payés de leurs rentes et agriers ${ }^{27}$ ". Dépôts

25. Il convient cependant de noter que cette " évidence " du caractère politique de la révolte nobiliaire est récente dans l'historiographie. Sur ce point, voir JouANNA, Arlette, Le Devoir de révolte. La noblesse française et la gestation de l'Etat moderne, 1559-1661, Paris, Fayard, 1989.

26. Platelle, Henri (éd.), Journal d'un curé de campagne au XVIre siècle, Lille, Presses universitaires du Septentrion, 1997, p. 102 et GUENARD, M., "Les gens de Verzy et VillersMarmery contre les Russes en 1814 ", Mémoires de la Société d'agriculture, commerce, sciences et arts de la Marne, 1905-1906, p. 67-74.

27. Arch. dép. d'Ille-et-Vilaine, 1 F 1655 et Bercé, Yves-Marie, Croquants et Nu-Pieds..., op. cit., p. 80 . 
publics ensuite : le cas de la Bastille, en juillet 1789, est trop connu pour que l'on y insiste ici.

Passons sur les armes de circonstance dont s'équipent certains lorsque gronde la révolte : J. Nicolas a bien décrit les " actes de rescousse villageoise " marqués par la prompte alarme de paysans accourrant " avec leurs bâtons et leurs outils, fourches, volants (faucilles), faux, fléaux, pioches, coutre de charrue, aiguillons, fouets aux lanières lestées de plomb ", quelque pierre saisie en chemin faisant parfois l'affaire ${ }^{28}$. On retrouvera pour une part les mêmes instruments face aux cosaques dans l'Est de la France en 1814 ou en 1906 pour s'opposer aux inventaires : à Verzy, dans la Marne, " chacun s'empare de ce qui tombe sous sa main : fusils, sabres, fourches, faux, etc. " pour faire face aux troupes russes qui s'avancent vers Reims durant la campagne de France tandis que, dans un autre contexte, les rapports de gendarmerie décrivent, dans l'Ille-et-Vilaine de la III ${ }^{\mathrm{e}}$ République, de " gros bâtons ", ferrés ou non, en certains cas " de la grosseur du poignet, dont l'extrémité était traversée de pointes longues " comme à Marpiré, rappelant la dimension utilitaire mais aussi symbolique de l'objet ${ }^{29}$.

La prise d'armes ne se limite pas à ces armes " par destination", comme le laisse entrevoir la mobilisation marnaise de 1814 évoquée plus haut, y compris lors d'alarmes a priori spontanées. Ainsi, en 1766, à Bais, paroisse rurale du diocèse de Rennes, dans un contexte marqué à la fois par de mauvaises récoltes et la libéralisation du commerce des céréales, une émeute prend pour cible des marchands blatiers venus du bourg voisin, Châteaugiron. S'ils sont assaillis par des hommes et des femmes " armés de bâtons, pelles, tranches, brocs " - à l'instar de la masse des accusés des justices seigneuriales qui, lorsqu'ils ne frappent pas à mains nues, utilisent avant tout ces objets du quotidien -, un tisserand, ancien soldat, est aperçu un "épée nue à la main ", ce qui contribue d'ailleurs à affirmer son statut de meneur, tandis que le maréchal-ferrant du village menace les intrus avec " des pistolets ${ }^{30}$ ". Pour anecdotique qu'il soit, le fait nous rappelle que si le port d'armes est un privilège nobiliaire, la possession d'armes est beaucoup plus commune, y compris, en France, avant la Révolution.

Des armes appartenant à la communauté des habitants tout d'abord, ainsi que le révèle, par exemple, l'étude des inventaires et des comptes

28. NicOLAS, Jean, La Rébellion française. Mouvements populaires et conscience sociale (1661-1789), Paris, Le Seuil, 2001, p. 75.

29. Simon, Jean-François, « Les armes de la violence paysanne. Faux et penn-baz: usages anciens et représentations contemporaines ", Kreiz, n ${ }^{\circ} 13,2000$, p. 151-168 rappelle la pérennité de cet objet jusqu'à l'après-Seconde Guerre mondiale dans les campagnes de Basse-Bretagne.

30. Arch. dép. d'Ille-et-Vilaine, 2 B 1865, Arrêt de la chambre du conseil du présidial de Rennes, 21 mai 1766. Sur cette émeute, voir LAGADEC, Yann, " Genre et mutations économiques et sociales : l'exemple de l'émeute frumentaire de Bais (1766) ", dans : BERGÈRE, Marc et CAPDEVILLA, Luc (dir.), Genre et événement. Du masculin et du féminin en histoire des crises et des conflits, Rennes, PUR, 2006, p. 69-83. 
des fabriques paroissiales bretonnes des XVe-XVII seconde moitié du XVI ${ }^{\mathrm{e}}$ siècle, celle de Louvigné-de-Bais, près de Vitré, acquiert à plusieurs reprises des armes, arbalète, épieux, lance, épée et autres salades - des casques - a priori peu utiles à l'exercice du culte, conséquence de la charge revenant aux paroisses bretonnes d'entretenir, depuis le $\mathrm{XV}^{\mathrm{e}}$ siècle, un et parfois plusieurs francs-archers et élus. Les traces de cet effort d'armement subsistent encore dans les inventaires du premier tiers du XVII ${ }^{\mathrm{e}}$ siècle : l'église abrite encore alors une "harquebuse à maiche " et " un viel espieu en forme de pertuissanne ", qualifié ailleurs de " hallebarde ${ }^{31}$ ". En 1481 déjà, à Chantenay, dans le diocèse de Nantes, les dépenses engagées par la fabrique pour son franc-archer représentent plus de $45 \%$ des " mises", des dépenses. Pour cette seule année de compte, il a fallu entre autres aux trésoriers de la fabrique faire " relever les brigandine du franc-archier ", payer " la motyié d'une jacquette ", "la moytié d'une paire de solers", acheter " une paire de brigandines neufves garmies de maheustres ", " une paire de chausse et ung pourpoint ", faire "fourbir les vouges, dagues et salades ${ }^{32}$ ".

À ces armes possédées collectivement, s'ajoutent celles détenues en propre par quelques-uns, malgré la tendance de l'État royal à réglementer de plus en plus strictement à compter des années 1660 non seulement le port d'armes, mais encore la simple possession. Ainsi que le révèlent, par exemple, les inventaires après décès bretons, les arrêts du Parlement visant à en réserver l'usage aux seuls nobles restent sans effets : les armes sont nombreuses dans les campagnes d'avant 1789 et, la chose mérite d'être notée, à parité chez les laboureurs et les autres, au moins en ce qui concerne les fusils, la possession de pistolets restant en revanche plus connotée socialement et réservée à la petite bourgeoisie rurale ${ }^{33}$. L'on dépasse donc ici le cas des voyageurs roturiers, marchands entre autres, autorisés à circuler avec une paire de pistolets dans leurs fontes. L'exemple n'est pas isolé : la rumeur prétend qu'il y aurait 300 fusils dans la seule paroisse de Saint-Marcel-en-Vivarais, en 1763, 200 à Milhaud près de Nîmes en 1785, pour certains achetés " au vieux fer chez les cloutiers " selon des témoins ${ }^{34}$. L'on est loin pourtant, en ces terres languedociennes, des frontières du royaume où, au XVIII ${ }^{\mathrm{e}}$ siècle encore, l'on n'hésite pas à armer les

31. Arch. dép. d'Ille-et-Vilaine, Louvigné-de-Bais G 33, inventaire des biens de la fabrique, comptes de 1611/1612 et 1613/1614. Voir aussi les quelques éléments donnés sur ce point par Restif, Bruno, La révolution des paroisses. Culture paroissiale et Réforme catholique en Haute-Bretagne aux XVI et XVII siècles, Rennes, Presses universitaires de Rennes, 2006 p. 45 .

32. La Nicollière-Tejeiro, Stéphane de, "Comptes de la fabrique Saint-Martin de Chantenay (1481-1506)", Revue de Bretagne et de Vendée, 1875, p. 180-184.

33. Sur ce point, LAGADEC, Yann, Pouvoir et politique en Haute-Bretagne rurale. L'exemple de Louvigné-de-Bais (XVI'-XIXe siècles), Thèse de doctorat en histoire, Université de Rennes 2/ Haute-Bretagne, 2003, p. 451 et 989.

34. Nicolas, Jean, La rébellion française..., op. cit., p. 411-414. Voir aussi, sur la place des armes dans la société d'Ancien Régime, CORVISIER, André, Armées et sociétés en Europe de 1494 à 1789, Paris, PUF, 1976, p. 19-20 et CORVISIER, André, L'Armée française de la fin du 
populations afin d'assurer la défense du territoire contre les incursions et autres descentes ennemies : ainsi en Alsace en août 1743, face aux hussards et pandours autrichiens, mais aussi de manière plus générale sur les littoraux, les miliciens gardes-côtes - au premier rang desquels les Bretons - se retirant chez eux avec leur armement.

Quelles armes? Et pour quoi faire? Ce sont donc là deux des questions auxquelles les contributions de ce dossier souhaiteraient fournir quelques éléments de réponse.

\section{Des campagnes de l'Ouest au Languedoc}

Il ne pouvait s'agir, à l'échelle de ce dossier, de traiter de l'ensemble des thématiques liées aux rapports entre port d'armes, prise d'armes et politisation. À travers des exemples localisés - Rennes, la Vendée, le Languedoc... - mais propres à ouvrir des réflexions plus globales, notre volonté était de privilégier quelques perspectives, les plus porteuses à nos yeux.

La première concerne la comparaison, l'étude simultanée des rapports entre prise d'armes d'une part, politisation des " élites " et politisation populaire d'autre part. Trop souvent en effet, les études sur la prise d'armes se focalisent sur une catégorie spécifique. C'est à un regard croisé sur les différentes strates sociales que nous invite ainsi la contribution de G. Aubert sur Rennes en $1675^{35}$. La seconde perspective touche aux rapports entre la prise d'armes et ce que l'on pourrait appeler une politisation conservatrice. En ce domaine encore, R. Dupuy a ouvert la voie à des réflexions qui demanderaient sans doute à être systématisées et étendues ${ }^{36}$. L'on connaît - assez - bien désormais en effet le rôle de la garde nationale dans l'acculturation "bleue ", républicaine et/ou libérale. Qu'en est-il cependant d'une politisation "blanche " et du rôle des résistances à la Révolution puis à l'Empire, à la monarchie de Juillet - voire à l'État et à ses représentants tout simplement? Le cas de l'Ouest de la France et, plus particulièrement, de la Vendée étudiée ici par A. Lignereux offre, en ce domaine, un cadre de réflexion particulièrement riche ${ }^{37}$. Les rapports entre politisation et prise d'armes contre l'ennemi extérieur, contre l'envahisseur constituent la troisième piste de réflexion. Les renouvellements récents des travaux sur la question du sentiment national - l'on pense, parmi de nombreux autres, aux ouvrages de D. Bell et E. Dziembowski sur la France, de L. Colley sur la

XVII siècle au Ministère Choiseul. Le soldat, Paris, Publications de la faculté des Lettres et sciences humaines de Paris, 1964, p. 53-72.

35. Voir, infra, AUBERT, Gauthier, «La prise d'armes rennaise de juin 1675 : une révolte civique?".

36. Dupuy, Roger, " Les paysans de l'Ouest et la duchesse (1828-1832). Considérations sur les aléas d'une acculturation politique de droite ", dans CroIX, Alain, LESPAGNOL, André et Provost, Georges (dir.), Eglise, Education, Lumières..., op. cit., p. 459-466.

37. Voir, infra, Lignereux, Aurélien, " La Première Restauration face à "l'inexplicable Vendée" : la levée de boucliers des 3-5 mai 1814 ». 
Grande-Bretagne ${ }^{38}-\mathrm{y}$ incitent tout particulièrement, notamment dès lors que l'on prend soin de dépasser le seul discours des "élites " pour s'arrêter sur les pratiques, celles des couches populaires, rurales notamment, mais pas seulement. C'est ce à quoi nous invite J. Hantraye, dans le cadre de la France de 1814, envahie par les armées coalisées ${ }^{39}$.

Bien évidemment, les textes qui suivent n'épuisent pas l'ensemble du sujet. Refuser de porter les armes dans certaines conditions participe ainsi, à n'en pas douter, d'une autre forme de politisation. Les débats autour de la place de l'objection de conscience dans les Treize Colonies devenues ÉtatsUnis tout au long du XVIII ${ }^{\mathrm{e}}$ siècle sont de ce point de vue très révélateurs; ils prennent d'ailleurs une nouvelle dimension dans les années 1787-1792, alors que l'on discute de l'adoption d'une constitution fédérale et des amendements définissant les conditions imposant le port d'arme aux citoyens pour la défense du pays, en contradiction donc avec les conceptions religieuses de certains, au premier rang desquels les Quakers ${ }^{40}$. Finalement, ici comme ailleurs, le rapport entre le statut d'homme libre, de citoyen et la possibilité voire l'obligation de porter les armes - je fais mienne les conclusions de V. Challet sur le bas Moyen Âge ${ }^{41}$ - apparaît de manière explicite. Il convient de prendre aussi en considération les liens, tout aussi évidents, entre résistances aux systèmes successifs de recrutement - milice d'Ancien Régime, levée d'hommes puis de conscription - et politisation, souvent réactionnaire en l'occurrence. J. Hantraye, dans sa contribution, évoque le cas du Nord, du Pas-de-Calais, du sud de la Belgique actuelle en 18141815 ; mais c'est aussi bien évidemment le cas en Vendée et en Bretagne en mars 1793, puis à nouveau en 1832. L'opposition à l'obligation légale de porter les armes passe ainsi, paradoxalement, par une prise d'armes, jugée quant à elle légitime : c'est en effet moins le service armé que l'on refuse, en bloc, que les conditions de ce service du pays par les armes, loin de chez soi, suite à un système de recrutement jugé par trop inégalitaire, rappelant d'ailleurs - paradoxalement - le tirage au sort de la milice d'Ancien Régime.

Les conditions dans lesquelles l'on dépose, l'on rend les armes après les avoir prises auraient sans doute mérité de plus amples développements : A. Lignereux nous pousse implicitement, dans son texte, à poursuivre la

38. Bell, David, The Cult of the Nation in France. Inventing Nationalism, 1680-1800, Cambridge, Havard University Press, 2001; Colley, Linda, Britons : Forging the Nation, 1707-1837, Londres, Pimlico, 2003 (2e éd.); DzIEMBowski, Edmond, Un nouveau patriotisme français, 1750-1770. La France face à la puissance anglaise à l'époque de la guerre de Sept Ans, Oxford, Voltaire Foundation, 1998.

39. Voir, infra, HANTRAYE, Jacques, " Les prises d'armes de 1814-1815, signe et facteur de la politisation des ruraux".

40. Voir par exemple, sur cette question, la mise au point de RENNER, Richard W., "Conscientious Objection and the Federal Government, 1787-1792 ", Military Affairs, 19744, vol. 38, p. 142-145, et les différences nettes entre les différents États fédérés, la tolérance du Rhode Island, du Massachusetts ou de la Caroline du Nord à l'égard des conceptions des Quakers s'opposant à la position plus rigide de la Pennsylvanie.

41. Voir, infra, CHALlet, Vincent, " “Al arma! Al arma!”. Prises d'armes et recours aux armes à l'époque médiévale : entre autodéfense et revendication de liberté ". 
réflexion en ce sens dans le cadre de l'Ouest des années 1814-1815. Les travaux récents de W. Bruyère-Ostells sur l'engagement des officiers de la Grande Armée dans les mouvements nationaux et libéraux après la chute de l'Empire illustrent aussi, pour une part, ce phénomène : les professionnels de la guerre qu'ils sont devenus au fil des campagnes proposent ainsi leurs services aux peuples d'Amérique du Sud en quête de leur indépendance, faisant leurs les combats des libéraux d'Espagne, de Grèce, de Belgique ou de Pologne, fomentant en France maints complots au cours de la Restauration, peuplant les sociétés secrètes à l'instar de la Charbonnerie, agissant plus ouvertement à la tête des insurgés lors des journées révolutionnaires de 1830 mais aussi de $1832^{42}$.

Enfin, la question - non exempte de provocation - de l'absence de tout lien entre prise d'armes, port d'armes et politisation nous semble pouvoir - et devoir - être posée. Le cas des clubmen, présents dans une quinzaine de comtés du Sud et de l'Ouest de l'Angleterre des années 1640, est de ce point de vue particulièrement intéressant. En effet, ils constituent à partir de 1645 des groupes armés parfois conduits par la gentry locale, réunissant plusieurs milliers d'hommes à l'occasion - 5000 environ dans le Somerset -, dont l'objectif est de se prémunir des exactions des soldats des deux camps : ni cavaliers ni parlementaires, leur caractéristique première aurait donc été le refus de la politisation en ces temps de guerre civile. Reste que les études menées au cours des trente dernières années sont venues largement remettre en cause cette vision par trop idéale de la situation : l'apolitisme de façade dissimulerait en effet une prise de parti évidente, pour un camp ou pour un autre en fonction des intérêts du moment ${ }^{43}$. Ne peut-on, cependant, trouver la marque de ce "degré zéro " de la politisation dans l'action menée par ces trois paysans bretons qui, attablés sans doute depuis de nombreuses heures déjà dans une auberge de Lancieux, au sud-ouest de Saint-Malo, en ce 6 septembre 1758, voyant passer un cavalier britannique conduisant " une vingtaine de chevaux anglais ", "formèrent le projet d'aller s'embusquer pour tâcher de surprendre quelques-uns desdits chevaux ", sans succès d'ailleurs ${ }^{44}$ ? Plus que politiques ou patriotiques, les motivations du coup de main, échafaudé dans les vapeurs d'alcool d'un cabaret, semblent strictement matérielles : la possibilité de s'enrichir facilement. Mais leur témoignage, recueilli dans le cadre d'une enquête pour trahison et espionnage, donne pour une part à leur modeste action de "résistance " une tout autre dimension, plus politique sans doute, aussi peu glorieuse qu'elle soit : ils ont pris les armes face à l'ennemi, alors même que la plupart des habitants fuyaient les environs,

42. BRuYÈre-Ostells, Walter, La Grande Armée de la Liberté, Paris, Tallandier, 2009.

43. UndERDown, David, " The Chalk and the Cheese : Contrasts among the English Clubmen ", Past and Present, ${ }^{\circ}$ 85, 1979, p. 25-48.

44. Lagadec, Yann et PerRÉon, Stéphane (avec la collaboration de David Hopkin), La Bataille de Saint-Cast (Bretagne, 11 septembre 1758). Entre histoire et mémoire, Rennes, Presses universitaires de Rennes, 2009, p. 177-178. 
que d'autres proposaient leurs services aux " envahisseurs " contre espèces sonnantes et trébuchantes.

On le voit : rien n'est simple en ce domaine de la politisation, notamment populaire. Tout est affaire de regard, de focale parfois, ainsi que pousse à le penser l'observation des sociétés plus contemporaines où l'on ne saurait juger de la politisation - ou non - des populations au regard du seul critère de la participation électorale. Des modalités diverses, spécifiques en fonction des contextes qui les voient éclore, hors de tout processus linéaire : telles sont d'ailleurs quelques-unes des conclusions des journées d'études sur le thème des rapports entre conflits et politisation ayant précédé celle du 9 janvier $2009^{45}$. Des conclusions que nous ferons nôtres.

\section{RÉSUMÉ}

La question des liens entre la politisation et la prise d'armes dans les sociétés des $\mathrm{XIII}^{\mathrm{e}}$-XIX ${ }^{\mathrm{e}}$ siècles n'est sans doute pas totalement neuve. Les historiens se sont cependant en général focalisés sur quelques moments-clés perçus comme particulièrement importants (la Révolution française, les mouvements populaires de 1830 ou 1848), privilégiant par ailleurs une politisation fondamentalement " républicaine ". Il nous a semblé qu'il était nécessaire d'affiner les questionnements sur les rapports entre la prise d'armes - légale ou non, encadrée ou pas - et la politisation - " progressiste " ou " conservatrice ", populaire ou non. C'est ce que se propose de faire ce dossier issu d'une journée d'étude tenue à Rennes en janvier 2009.

\section{ABSTRACT}

The question of the relationship between politicization and taking up arms in the societies of the $13^{\text {th }}-19^{\text {th }}$ centuries is probably not a new one. However, historians have generally concentrated on a few key moments, seen as particularly important (the French Revolution, the popular movements of 1830 or 1848), also laying stress on a fundamentally "republican" politicization. For us, it was necessary to refine the questions bearing on the relationship between taking up arms - legally or not, in an organized way or not - and politicization, be it "progressive" or "conservative", popular or not.

45. Bourquin, Laurent et HAmon, Philippe (dir.), La Politisation. Conflits et construction du politique depuis le Moyen Âge, Rennes, PUR, 2010. 